\title{
WIKA: A Projected Augmented Reality Workbench for Interactive Kinetic Art
}

\author{
Yunwoo Jeong, Han-Jong Kim, Gyeongwon Yun, Tek-Jin Nam \\ Department of Industrial Design, KAIST \\ 291 Daehak-ro, Yuseong-gu, Daejeon 34141, Republic of Korea \\ \{nnitgd, hanjong.kim, heoragi53, tjnam\}@kaist.ac.kr
}

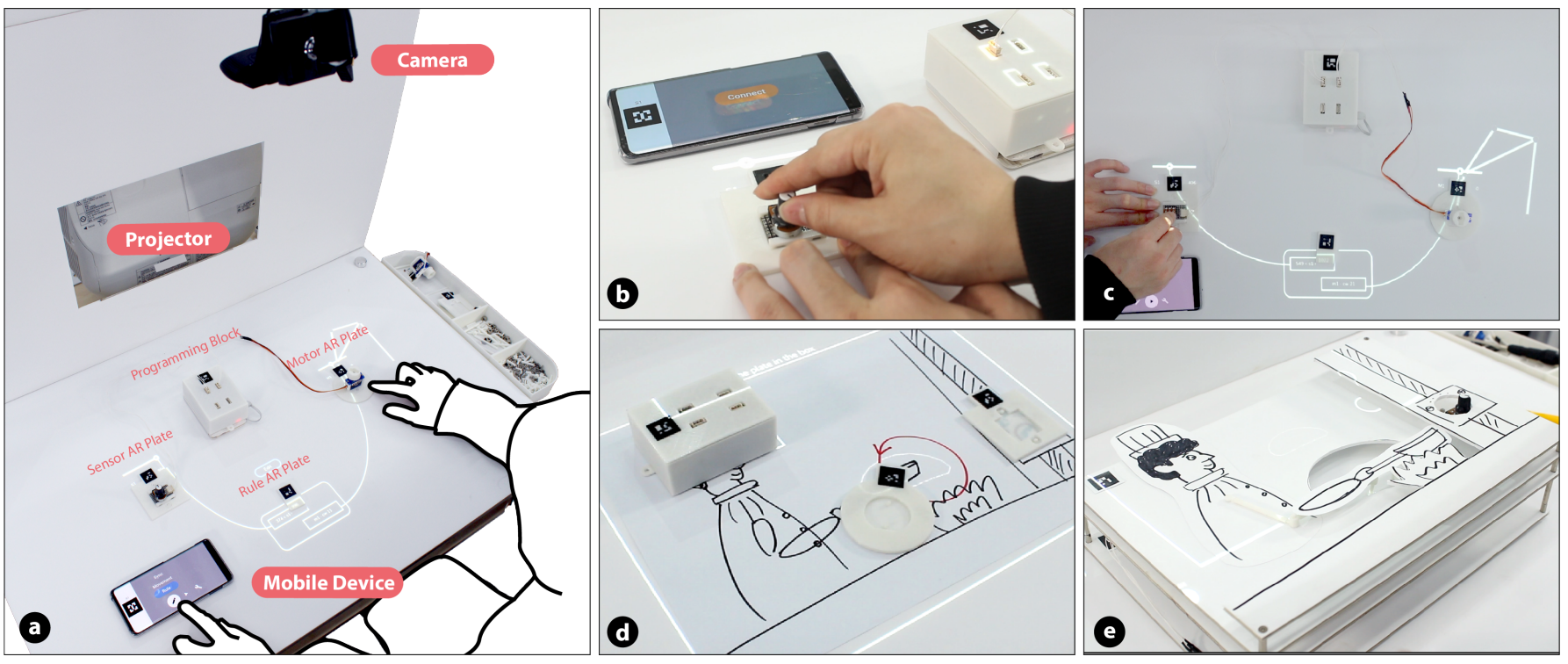

Figure 1. WIKA supports the whole creation process of the interactive kinetic art (IKA) (a) A projected AR system with a mobile device efficiently makes an interactive tabletop. (b) Physical components are applied from the initial phase of prototyping. (c) The projected information connected with physical components enables the programming and simulation on the workspace. (d) Tangible objects support a trial-and-error process during the making phase. (e) The user can build the IKPF with generated guideline.

\begin{abstract}
Iterative artistic exploration, mechanism building, and interaction programming are essential processes of prototyping interactive kinetic art (IKA). However, scattered tools and interwoven workflows across digital and physical worlds make the task difficult. We present WIKA, an integrated environment supporting the whole creation process of IKA in the form of a layered picture frame in a single workspace. A projected AR system with a mobile device efficiently makes an interactive tabletop. The projected information connected with physical components (e.g. sensors and motors) enables the programming and simulation on the workspace. Physical components are applied from the initial phase of prototyping
\end{abstract}

Permission to make digital or hard copies of all or part of this work for personal or classroom use is granted without fee provided that copies are not made or distributed for profit or commercial advantage and that copies bear this notice and the full citation on the first page. Copyrights for components of this work owned by others than the author(s) must be honored. Abstracting with credit is permitted. To copy otherwise, or republish, to post on servers or to redistribute to lists, requires prior specific permission and/or a fee. Request permissions from permissions@ acm.org.

UIST '20, October 20-23, 2020, Virtual Event, USA

(C) 2020 Association for Computing Machinery.

ACM ISBN 978-1-4503-7514-6/20/10 \$ \$15.00.

http://dx.doi.org/10.1145/3379337.3415880 using an AR plate, and this supports the iterative trial-and-error process by bridging the workflow. A user study shows that WIKA enabled non-experts to create diverse IKA with their ideas. A tangible interaction and projected information enable the iterative and rapid creation. The method that integrates the hardware and software in the physical environment can be applied to other prototyping tools that support the creation of interactive and kinetic elements.

\section{Author Keywords}

Prototyping; Augmented Reality; Interactive Kinetic Art; Tangible Interface; Augmented Information; Visual

Programming

\section{CCS Concepts}

-Human-centered computing $\rightarrow$ Mixed / augmented reality;

\section{INTRODUCTION}

An interactive kinetic art (IKA) is commonly created by makers. The IKA incorporates movement and interactivity. It is changed according to human behavior and the environment 
using sensors and motors. In the creation process of the IKA, it is necessary to consider engineering, computational, and artistic aspects altogether. It is difficult to design and make the IKA for non-experts without any related skills or experience with engineering, programming, artistic exploration, and hardware making [41].

One of the most challenging aspects of creating the IKA for non-experts is dealing with various elements, such as physical I/O components, programming interactivity, and mechanical parts, during the creative exploration. Many tools and workflows scattered around physical and digital environments increase the difficulty of the creation. After designing and simulating prototypes in the digital environment, unexpected problems are found in the physical environment. The gap between physical and digital environment makes users spend a lot of time finding and trying to resolve the problems.

Prior tools have been introduced to support the creation of the IKA or other similar tasks $[4,7,42,38,27,35]$. These tools enabled rapid creation with tangible exploration, for example, using a modular block system. Although a few simulation tools $[1,21,39]$ have been suggested, there is a lack of research examining an integrated prototyping environment that allows the iterative trial-and-error process to achieve the desired results.

In this paper, we present WIKA (Workbench for Interactive Kinetic Art, Figure 1), an integrated environment that offers a whole creation process and elements of the IKA. It allows non-expert users to create the IKA in the form of a layered picture frame with iterative trial-and-error process. To support the whole creation process for non-experts and to minimize the gap between the physical and digital environment, we adopted a tangible interface and projected AR visualizing diverse information promptly and interactively during the creation process. A mobile device efficiently supports the interface according to the situations. A sensor value, motor speed, logical relation, and simulated linkage mechanism, are overlaid onto the corresponding hardware. This method enables defining, modifying, and testing the logical relation between sensors and actuators in the workspace. Physical components are involved from the beginning of the creation process. AR plates connect the physical components and system. A user study of prototyping an interactive kinetic picture frame (IKPF, Figure 1e) as an example of the IKA showed that the integrated environment of WIKA allows non-experts to rapidly create the diverse IKA based on their own design ideas.

The contributions of this paper are threefold. First, it presents a novel integrated prototyping environment for supporting the whole creation process of the IKA, which supports the artistic exploration, mechanism building, and interaction programming in a single workbench. Second, it suggests a visual programming method using projected AR that defines and tests the logical relation using physical components, and then it represents the invisible information with corresponding hardware in the physical environment. Finally, the user study demonstrated that the proposed system enables a top-down approach for creating the IKPF by guiding the position of the sensor, motor, mechanism, and trajectory on the idea sketch.

\section{RELATED WORKS}

\section{Prototyping Tool for Movement and Interactivity}

For non-experts, creation with movement and interactivity is difficult because various skills are required. Several works have presented tools that support the creation of kinetic papercraft. FoldMecha [33] applied the parametric design approach for designing the linkage mechanism. CardBoardiZer [49] generated the articulated paper model from the 3D mesh model. MoveableMaker [3] helped to design and create the physical interaction of papercraft with a mechanism template. Some works applied electronics to the papercraft. Lo et al. presented the tool for creating aesthetic electronics by combining the circuits with drawing [30]. PEP [34] helped to create the electronic device by inserting thin circuits into the layered paper. PaperPulse [39] supported the interactive papercraft with circuits and electronics in the paper. These tools supported the movement and interactivity of the craft with low cost material. Some works have supported the fabrication with a $2 \mathrm{D}$ blueprint. However, there is still a gap between displayed or printed blueprints and the physical creation process.

Several studies have focused on the framework or algorithm to generate the mechanism from desired movement $[45,31,8,6$, 50, 32]. Robiot [29] also applied a generative design approach for actuating the existing object from an iterative movement input. The generative design approach enables a user to design the desired movement with an intuitive interface. Prior works have supported the creation of craft, including movement and interactivity, with various methods. WIKA also supports the creation of movement and interactivity. On the other hand, we focused on the integration of physical and digital environments for incorporating scattered tools and workflow rather than methods for generating the desired outcome.

\section{Tangible and Visual Programming Tool}

To support the programming behavior of non-experts, several tangible programming methods have been presented. Horn et al. developed a block-based tangible programming method that can be used in class [14]. StoryBlocks [26] enabled the user to create audio stories with tangible programming. CreaTable [29] is a tangible programming tool that enables the creation of multimedia contents with various tangible objects that contain specific meanings. MakerWear [18] helped to create interactive cloth by attaching a modular electronics. Cassinelli and Saakes [5] introduced the tangible programming approach with proxemic interaction and a data-flow method. The tangible programming enables the user to create the outcome without learning the programming language. The exploration with tangible objects is directly connected to the outcome. We apply a tangible interface with physical components for programming and testing in the physical environment.

Meanwhile, a visual programming method has been applied in several works. Scratch [40] is one of the most popular visual programming tools using a block-based interface. TriggerAction-Circuits [2] provided a visual programming method that forms a relation between inputs and outputs by connecting the wires. Leonardi et al. [28] presented a tool for defining robot behavior with a hierarchical text block selection. The visual programming method helps to easily figure out the 
overall situation. However, connectivity with hardware is lower than tangible programming approaches. We apply a visual programming with projected AR with a mixture of tangible and visual programming approaches. Our system supports the programming in the AR environment by allowing the user to directly test the components with the tangible interface while checking the augmented visual information.

\section{Interactive Tabletop and Augmented Reality System}

Several researchers have studied the method for bridging the physical and digital environments. Interactive tabletop systems have been used for interactive simulation. URP [46] is an interactive tabletop system that supports the lighting simulation by moving tangible objects. Tangible 3D Tabletops [9] is an urban planning simulation tool that makes a new projection surface with tangible objects. miniStudio [22] applied the interactive AR system with an invisible marker for large space scenarios. These works support the interactive simulation with the augmented information that follows the movement of tangible objects. However, they focused on the simulation of the contents rather than making physical outcomes.

In the fabrication area, some researchers proposed a prototyping tool that helps users design and edit the components in the physical environment. CopyCAD [10] is an augmented reality system that is combined with the $\mathrm{CNC}$ machine. It allows novice users to copy the physical parts, edit the geometry, and engrave with the machine. MixFab [48] is a novel mixed-reality environment that supports 3D modeling with physical objects and a gesture-based interface. VirtualComponent [25] combined the virtual and real electronics in the mixed reality system for rapid circuit construction and testing. Prior works have enabled the iterative trial-and-error process in the physical context by bridging the physical and digital environments. However, they only focused on the static elements. Our system expands the application area to support the interactive and kinetic elements.

Several works have studied the interface of the tabletop environment with tangible objects. ReacTable [16] provides a music creation interface by moving tangible objects. Tangible Bots [36] provides an interface that visualizes information with the groupings of the actuated tangible block. Suzuki et al. [44] explored an interaction method for controlling the behavior of small robots in a projection AR system. ARcadia [19] supports the creation of the tangible interface with an AR-based platform and papercraft objects. In previous studies, tangible objects have been used as an interface element for conducting a specific function. In our work, we expanded the role of tangible objects as a medium that connects the workflow of the whole reation process while also supporting the creation of the desired outcome by providing augmented information.

Several works have applied the mobile AR system to enhance the activity of users. Smarter Objects [13] helps users extend the functionality of existing objects with AR technology. Affinity Lens [43] is a mobile AR system for the affinity diagram with a diverse analysis method based on textual data. G-raff [20] is a smartphone mounted block device that supports a spatial interaction on the tabletop. These systems enhance user activities by providing visual information bridged with the physical environment. We also apply the mobile device as an interface of WIKA, but in addition to providing information, it is used as an adaptive interface that connect the users and the components of the projected AR system.

\section{WIKA: WORKBENCH FOR INTERACTIVE KINETIC ART}

WIKA is a projected AR workbench that allows non-expert users to create IKA in the form of a layered picture frame. We defined this particular form of the IKA as the IKPF. The IKPF contains the sensors and motors, and the logical relation among sensors and motors, which we called Rule, should be defined. The projected AR system supports an iterative prototyping process of the IKPF, including design, programming of the rule, simulation, and physical creation in a single workspace. To support the integrated prototyping environment and workflow, we used the AR plate, a 3D printed plate with an AR marker. Physical sensors and motors are assembled with AR plates to be used as a tangible interface for defining the rules. Invisible information, such as sensor value, motor speed, defined rule, simulated mechanism, and trajectory, is augmented and immediately reflected on the workspace. AR plates are also used for seamless connection for physical creation. Sensor, motor, mechanism, and trajectory can be positioned by placing the AR plates onto the idea sketch. The system generates the one-to-one scale guideline based on the position of AR plates.

\section{Hardware Configuration and Components}

\section{Workbench and Mobile Device}

WIKA consists of a projector, a camera, a mobile device, and a workspace. It allows the user to create the IKPF with an AR marker attached to the hardware in the workspace. The AR marker is detected by the camera and the related information is displayed above the hardware through the projector. For an unobtrusive, familiar, and cost-effective interface, we chose the mobile device as an input interface. The mobile device makes the localized touch area to control the function in the workspace. It enables the flexible change of interface according to the user's intention. The user can easily access the system through the Web application without additional devices. The AR marker on the mobile device is used for position tracking and duplicating the graphics of the workspace on the mobile screen (Figures $2 \& 3$ ).

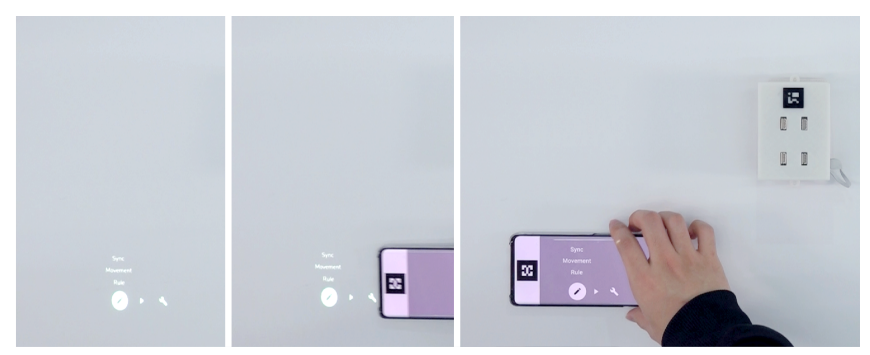

Figure 2. The main menu located at the left bottom of the workspace. The user can activate the main menu by placing the mobile device in the same location. The user can change the mode in the main menu. The main menu consists of a Edit (a Sync, a Movement, and a Rule), a Simulate, and a Fabrication. 


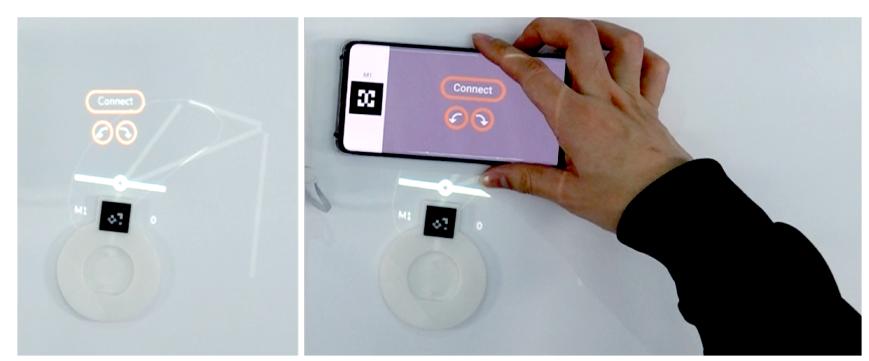

Figure 3. Interface buttons of AR plates can be activated with the mobile device.

\section{AR Plate, Programming Block, Sensor, and Motor Unit}

AR plates with an AR marker are used for augmenting invisible information and connecting the workflow. There are three types of AR plates: sensor, motor, and rule. The detected AR plate is activated on the workspace and it can be deactivated by flipping or removing from the workspace. Interface buttons and augmented information are located above the AR plate. Interface buttons can be pressed with activation with the mobile device. Sensor and motor AR plates hold sensor and motor units. Rule AR plates are used to define the rules between sensors and motors. The programming block supports the physical I/O of components and wirelessly communicates with the server. The programming block has an AR marker for position tracking, and sensor and motor units can be connected with an augmented guide.

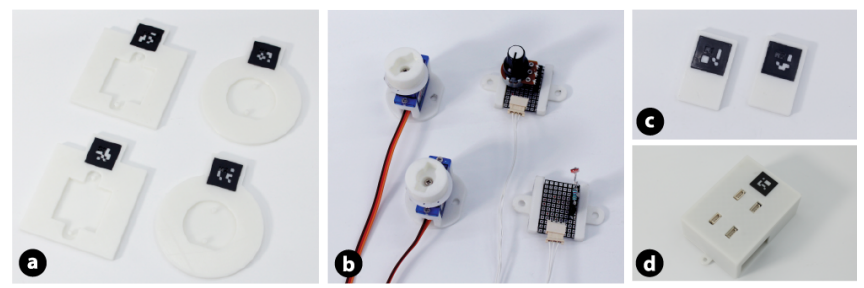

Figure 4. (a) Sensor and motor AR plates. (b) Motor (left) and sensor(top: knob, bottom: light sensor) units. (c) Rule AR plates. (d) Programming block.

\section{Foam Board, Support Pillar, Plastic Tube, Joints, Fasteners,} and Stationery

There are additional materials to create the IKPF. The IKPF has a layered structure with four layers of foam boards. To display the making guideline, the AR marker is attached to the foam boards. Layered foam boards are supported by support pillars. The linkage mechanism, which is one of the IKPF's components to represent movement, can be made by cutting a plastic tube and connecting it with 3D printed joints. The linkage mechanism, sensor unit, motor unit, and prototyping board can be attached to the plate by piercing a hole in the foam board and assembling with fasteners. We provided the stationery for assembly and other artwork.

\section{Usage Scenario: Creation of the IKPF}

To illustrate the usage scenario of WIKA, we introduce how a user creates the IKPF. Thie IKPF consists of a gas valve and chef. When the gas valve is rotated to the right, the chef starts cooking by shaking the pan. As the gas valve is rotated more, the chef moves the pan faster.

\section{Ideation and Sketching}

The user sketches the component, movement, and interaction (Figure 5). The user draws the chef, gas valve, and gas stove. The user wants to represent the gas valve with the knob and connect the pan and arm with the linkage mechanism for generating the movement. The user plans to place the body of the chef in the background and the gas stove in the foreground.
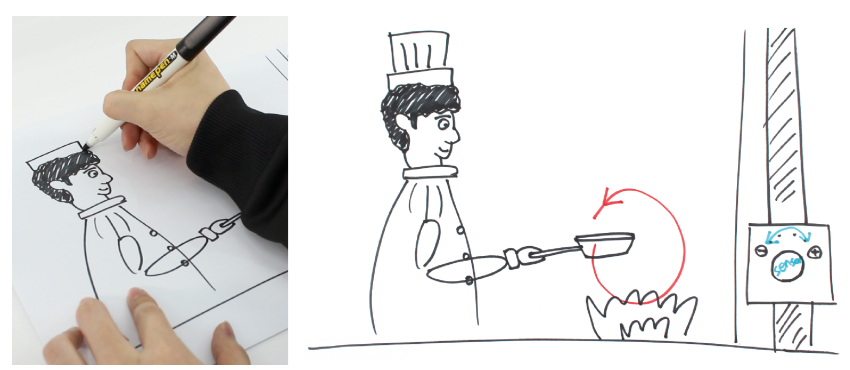

Figure 5. The sketch of the IKPF. The user draws the components and annotates the interaction.

\section{Sensor \& Motor Testing and Connection}

The user can check the connection location in the programming block in sync mode. The user places a sensor and motor AR plate and activates the Connect button with the mobile device. Then, the connection location is displayed on the programming block. After connection, the user can check the sensor value with the displayed value (Figure 6a) and motor rotation by pressing the buttons (Figure $6 \mathrm{~b}$ ).

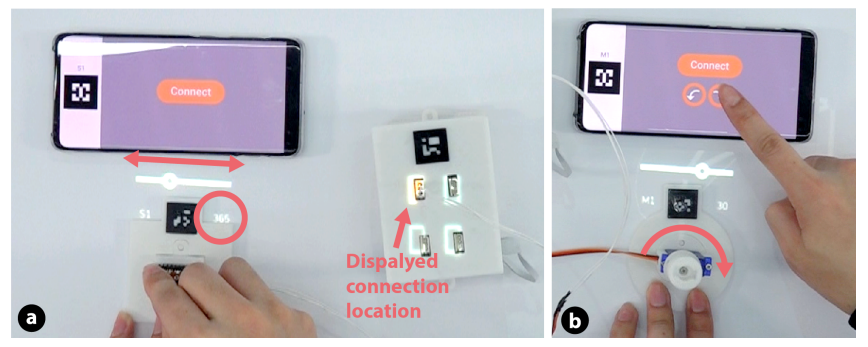

Figure 6. (a) The connection location is displayed on the programming block. Change of the sensor value is immediately reflected in the workspace. (b) Testing the DC motor with the mobile device.

\section{Programming the Rule}

The user then sets a rule, a relation between the sensor and the motor in rule mode. The user places a rule AR plate on the workspace and activates the Edit button above the rule AR plate with the mobile device. With the (+) button above the sensor and motor AR plate, as shown in Figure 7a, the user can select the desired sensors and motors for programming. The sensor value range can be defined by controlling the connected sensor (Figure 7b). When the user presses the value box, the sensor value is reflected in the value box. To set the value, the user has to press the value box again. The user can set the direction of the motor as clockwise (CW), stop, and counterclockwise $(\mathrm{CCW})$, and the speed ranges from 0 to 100 (Figure 7c). Once the rule editing is done, the user can save the rule by pressing the Apply button above the plate. Then, the defined rule is displayed on the rule AR plate (Figure 8). The user sets the sensor value range from 550 to 800 , the motor direction to $\mathrm{CCW}$, and the motor speed to 20 . 


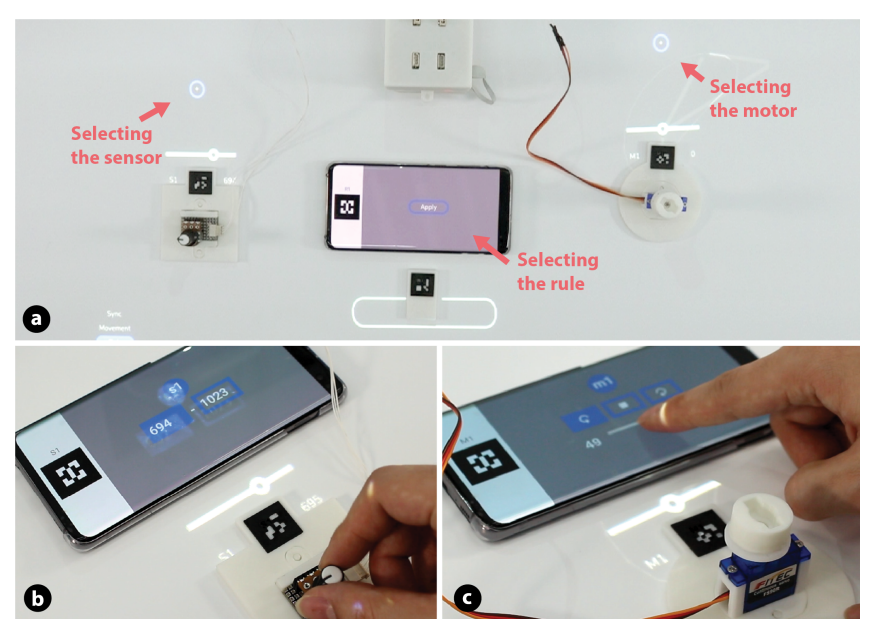

Figure 7. Programming the rule by (a) selecting the rule AR plate, (b) setting the sensor range, and (c) setting the motor direction and speed.

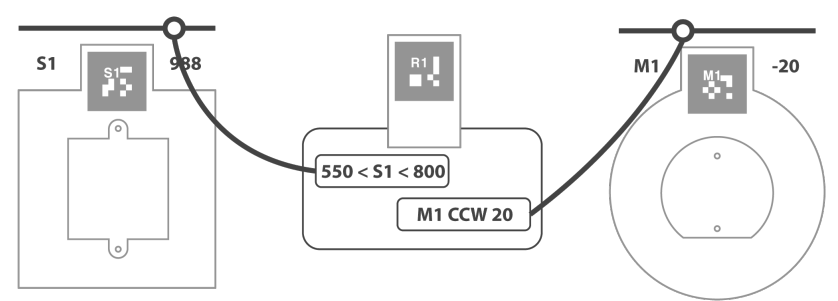

Figure 8. A diagram of the programming result. The left column is sensor conditions and the right column is motor conditions. The sensor conditions contain the name and range of the sensor value. The motor conditions contain the name, direction, and speed of the motor. Each component is connected with the corresponding $A R$ plate.

\section{Simulating, Modifying, and Adding the Rule}

In simulation mode, the user can simulate the defined rule with a physical sensor and motor. The user simulates the rule by controlling the sensor while examining the sensor value, defined rule, and motor actuation. The rule can be edited using the same process of rule definition. Because the motor speed is high, the user activates the edit mode and changes the motor speed to 10 . To represent the situation of rotating the gas valve further to the right, the user adds a second rule AR plate. The user deactivates the first rule by flipping the first rule AR plate. The user sets the sensor condition from 800 to 1023, the motor direction to CCW, and the motor speed to 30 , and the user simulates the second rule. After simulation and modification, the user activates both rules and simulates the two rules at the same time

\section{Positioning the Components and Movement Design}

The user can start the physical creation with guidelines in fabrication mode. The user places the AR plates by considering the component position and trajectory of movement with sketches (Figure 9a). The mechanism can be rotated by rotating the motor plate and resized with the Scale down \& Scale up button (Figure 9b). The user rotates and resizes the mechanism while checking the trajectory of the movement. After that, the user puts the programming block in the empty position to position it. To store the position of the components, the user presses the Capture button in the mobile device.

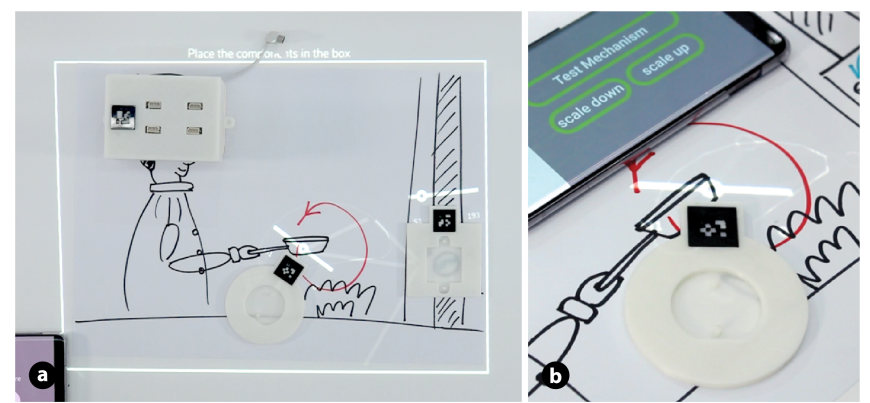

Figure 9. (a) Positioning the components with the AR plates. (b) Changing the movement with the motor $A R$ plate and a mobile device.

\section{Making with Real Scale Guide}

After storing the position of the components, the user can make the IKPF with the projected guideline. When the user places the foam board on the workspace, WIKA displays the real scale guideline about the component and hole position. There are four foam boards to be layered. The first layer holds the motor unit and programming block. The user can find the position of the motor unit and programming block with the augmented guideline (Figure 10a). The second layer holds the linkage mechanism. The user can mark and cut the plastic tube with the displayed mechanism (Figure 10b). After that, the user assembles the linkage mechanism with the joint and connects it to the foam board. The third layer holds the sensors, the path for the movement, and the background picture. To make the hole for the mechanism, the user marks a path bigger than the displayed trajectory and cuts it out (Figure 10c). The user pierces the hole and assembles the sensor. As the component position and size are changed, the user redraws the background (chef body) and the moving object (arm \& pan). The fourth layer holds the sensor hole and the foreground picture. The user redraws the gas stove and gas value on the foam board (Figure 10d) with the real position of movement and the sensor and cuts it out. Lastly, the user assembles the four layers with support pillars and attaches the pictures. The user then tests the first and second rules together.

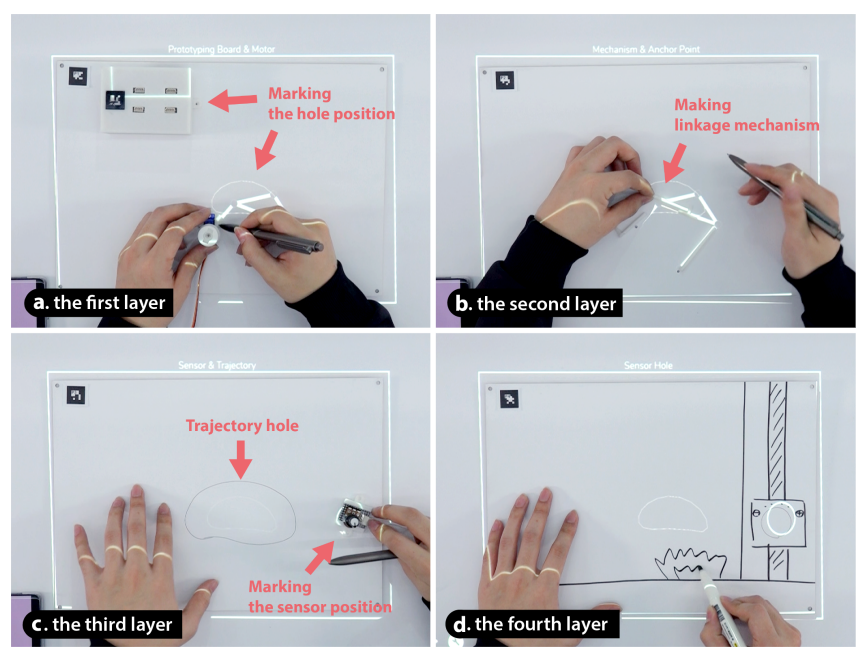

Figure 10. Summary of the making process. (a) Marking the component holes position and assembling. (b) Making the linkage mechanism. (c) Marking the path of the trajectory hole and assembling the sensor. (d) Redrawing with the displayed guide. 


\section{Iterative Test and Modification}

After assembling, further modification of the rule can be conducted with the AR plates and the mobile device on the workspace while testing the IKPF (Figure 11).

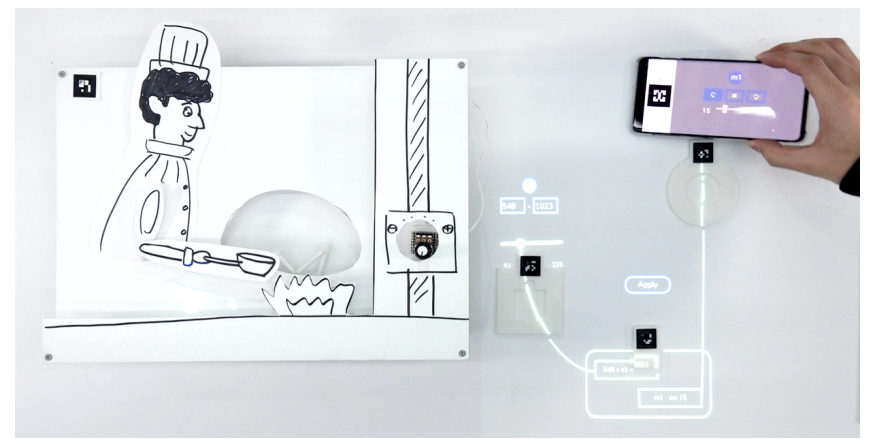

Figure 11. After assembling the components, it is still possible to edit the rule using the AR plates and the mobile device.

\section{Implementation}

Three Web-based applications (mobile, camera, and projector) are implemented with a single Express server and multiple .ejs view engine packages of Node.js. The Express is a framework for operating a Web server, and the ejs view engine renders a Web document. The interface of each application is developed with an ejs view template using HTML and JavaScript. All applications (mobile, camera, and projector) have operated on a single server and been deployed on the public Web browser. The Web server, camera application, and projector application are executed in the main computer. The camera application sends the data including the position and rotation of the detected AR marker using AR.js library. The projector application displays the changed information to the workspace through the projector. The mobile application is accessed in the mobile device and displays the interface buttons by receiving data of the closest AR marker.

The programming block consists of Raspberry Pi 3 for server communication and Rpino (an Arduino-compatible board) for extending the physical I/O. It is covered with a 3D printed case, and pin connectors were installed for easy connection. A node.js-based daemon was installed on the Raspberry Pi 3 for communication with the Web server and the hardware parts. The programmed rule is converted to the JavaScript code that exports the execution of motors according to the sensor value. Johnny-five API is applied to control the hardware with JavaScript language. All data of WIKA are shared via socket.io stream through the Web server. The virtual linkage mechanism design and simulation are implemented using the source code of the M.Sketch [21] with three.js. The other augmented information was displayed using $\mathrm{d} 3$.js. The position of augmented information was calibrated with the coordination of displayed four AR markers and perspective transformation after installation of the camera and projector.

\section{USER STUDY}

We conducted a user study to identify that WIKA enables the rich creation of the IKPF and integrates the environment and workflow effectively. The detail questions of user study included the following: (1) Is the WIKA capable of creating

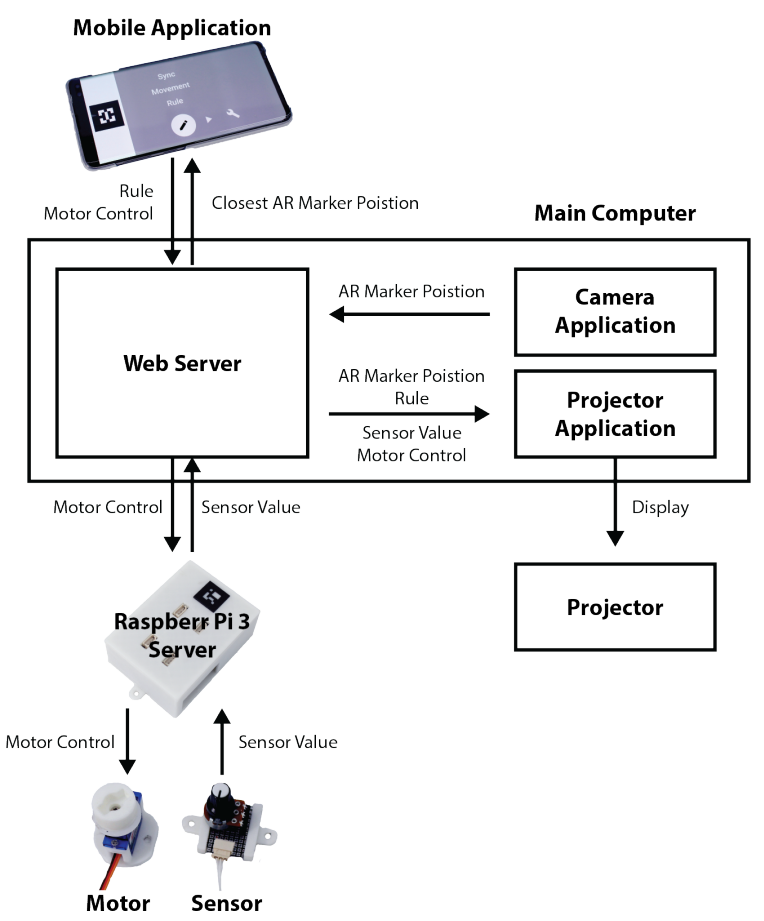

Figure 12. Software configuration and data stream diagram of WIKA. Every application communicates via a socket.io stream through the Web server

the diverse IKPF? (2) Does WIKA support the whole creation process? (3) How does the tangible interface and augmented information affect creation activities? (4) What are the improvement points?

We recruited ten undergraduate students in the campus (7 males and 3 females, ages $18-23, \mathrm{M}=20.4, \mathrm{SD}=1.65$ ). No participants had any experience in controlling hardware, such as sensors, motors, or linkage mechanisms. In the pre-survey, participants responded that they enjoyed making something $(\mathrm{M}=4.1, \mathrm{SD}=0.74,5$-point Likert scale) but had never experienced making this kind of the IKA.

The participants were asked to build the IKPF, expressing dynamic activities or objects of their interest. We explained that the IKPF can be used as an exhibition or signage in a shop where related items are sold and to draw customers' attention. To minimize the task time, we allowed participants to use a maximum of two sensors and two movements. There were two types of sensors (a knob and a light sensor) and one type of movement (circular movement with four-bar linkage mechanism). The knob ranged from 0 to 1023 and the light sensor ranged from about 200 (when the sensor is covered by a finger) to about 800 (when light strikes the sensor).

The procedure of the user study is as follows. First, we conducted five minutes of pre-interview to clarify the physical computing knowledge level of participants. Then, we introduced the overall function of WIKA and an example of the IKPF for 5 minutes. After a short introduction of the task, participants had 10 minutes for an ideation session. They sketched the outline of component location, movement, and interaction with the identical size of the outcome. After the 

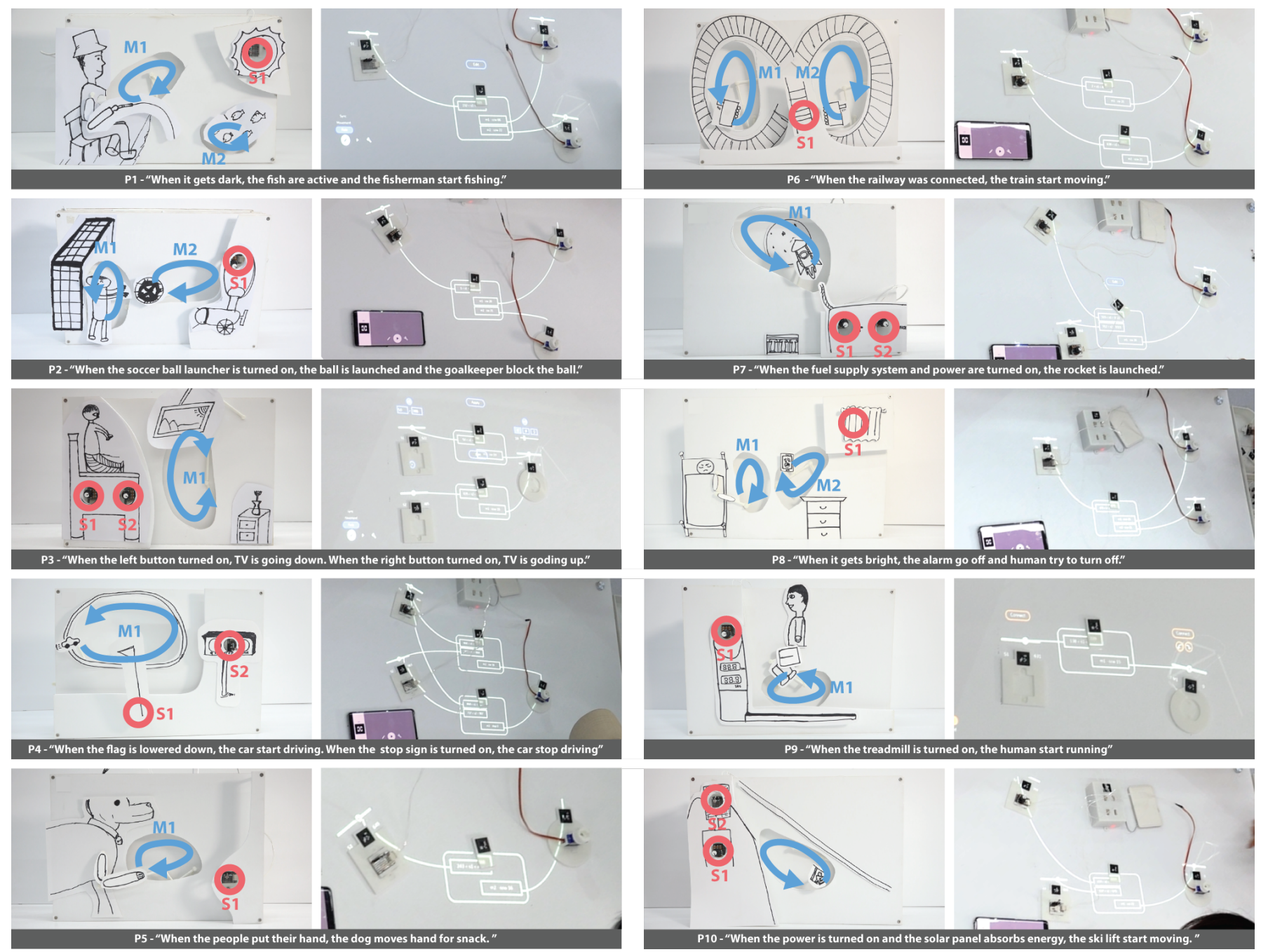

Figure 13. The results of the user study. The picture on the left is the interactive kinetic picture frame built by participants. The blue lines are the movement that was built with the four-bar mechanism and the DC motor. The red circles are sensors. The picture on the right is the final result of programming. P1: A fisherman and fishes. P2: A goalkeeper and a soccer ball launcher. P3: A person controlling the height of a TV. P4: A car, a flag, and traffic lights. P5: A dog. P6: Two trains waiting for a rail change. P7: A satellite rotating around the moon. P8: A person who wants to turn off the alarm. P9: A runner and a treadmill. P10: A skier and ski lift.

ideation session, participants worked on the main task for a maximum of 40 minutes. During the making phase, we aided in assembling and making holes to allow participants to fully concentrate on marking the component position and redrawing with augmented information. After completing the main task, we conducted a semi-structured interview about the effectiveness and improvement points of WIKA. The interview was conducted for 20 minutes.

\section{Design Outcome}

All participants successfully created the IKPF within the short evaluation session (Figure 13). Among the ten participants, four participants( $\mathrm{P} 3, \mathrm{P} 4, \mathrm{P} 7$, and $\mathrm{P} 10)$ used two sensors and one motor, four (P1, P2, P6, and P8) participants used one sensor and two motors, and two participants (P5 and P9) used one sensor and one motor. The participants made a scenario involving sensors even though there were only two types of sensors.
The participants represented various ideas with the circular movement. P4 and P6 attached the drawing on the knob to represent the physically linked scenario. P8 hid the light sensor behind the curtain drawing to represent the situation of the morning. P5 bridged the IKPF and real world by making the interaction between the dog and the hand. Most of the scenarios were implemented with one rule. In these scenarios, drawings are continuously rotated according to the sensor condition. Some participants (P3, P4, and P6) used two rules to create a different situation. The scenario of $\mathrm{P} 3$ was unique because the position of the object was controlled by movement. The TV is going up and down according to the sensor value. P4 made two situations. The motor is actuated when the flag (S1) is lowered down, and the motor stops when the light sensor detects the light. P6 also made two situations. Each motor should be actuated according to the different sensor conditions. 


\section{Observation and Participant Feedback}

The single workspace of WIKA, which supports the creation activity including the mechanism, programming, and artwork, received positive comments from participants. The whole creation process is bridged with augmented information, AR marker embedded AR plates, and the mobile device. From the initial phase of design, the participants developed their ideas together with the hardware. Most participants commented that the definition of sensor value with the real sensor was intuitive. They found the appropriate range of sensor values without any explanation about the sensor value. The rule plate helped them manage the rule in the workspace. P4 said, "Manually operating (rule AR plate) by flipping and controlling felt familiar and natural." After programming, augmented information helped them simulate and test the rule with real corresponding sensors and motors. The participants commented that overlaid visual information was helpful in checking the overall situation and testing the rules.

In the initial phase of making, it was possible to rapidly move on to the next step with the sensor AR plate, motor AR plate, and programming block. The participants determined the position of the sensor and mechanism by placing the AR plates on the sketch. P5 commented on the workflow connection, "Making was easier by using hardware that I have been operating from the beginning." The real scale guideline enabled the participants to place the components and redraw the picture by considering the location and size. P10 said, "At first I was not sure about the component position, but the consideration of the components made pictures to change a lot." The displayed guideline enabled them to find the position of the component in each layer. Rule modification was allowed with plates after assembly. P7 said, "During the editing state, I thought I had to disassemble it, but rather, it was convenient since editing was possible without it."

Meanwhile, the localized input interface, which supports the programming interface in the workspace, received positive comments from participants. The participants mentioned that the mobile device simplified the interface by activating the interface with one plate. P4 said, "It might be inconvenient to touch several buttons but, it made easy to understand by operating the features on the mobile device by selecting desired devices only." P1 also commented, "Even without knowing the hardware operational method, its function is shown to directly guide what should be done in hardware."

The participants mentioned that the creation process with a visual programming method using projected AR lowers the barrier. P8 said, "It was easy to understand because when making and connecting rules using hardware, visual information visualized big pictures while detailed works such as connecting wire were abstracted." P3 said, "It was easy to learn the concept by accepting hardware-orientation in ahead." Some participants commented that it was easy to make thinking into a logical relation without a complex process. P10 said, "I was able to proceed with my thinking process without considering the grammar." P5 said, "Delivering my imagination into realization by actually moving and expressing hardware helped to understand it better."
Most of all, they actively engaged in creating the IKPF with WIKA. During the pre-interview and ideation session, the participants worried about making the IKPF because they didn't have experience with it. However, WIKA increased interest in the creation of the IKPF. Some participants took the video to share the result with other people.

\section{DISCUSSION AND FUTURE WORK}

WIKA provided a single workspace that supports the whole creation workflow with integrated software and hardware. Physical sensors and motors were applied from the beginning of the workflow. Logical relation between sensors and motors was efficiently defined in the physical environment with the physical components. Manipulating physical AR plates supported the interface more acceptable. Changes of sensors, motors, and rules are immediately reflected in the workspace. Workflow is connected to the making phase in the single workbench with the same physical components. This feature facilitated the top-down approach for the assignment while supporting rapid and reflective workflow. It allowed the user to build the outcome while testing the defined rule. Nonexperts need creative tools that allow the top-down approach and that are focused on the outcome of activity, even without a concrete concept [47]. The approach can be adopted to other applications, such as an end-user customized intelligent system or robot programming. Interactive and kinetic elements can be effectively built in the physical context.

For a seamless integration of environment and workflow, further research about the interaction technique in the workbench can be explored. At first, the detection technique could be improved for effective recognition of the physical environment. The AR plates might be unnecessary if the system can precisely detect the sensors and motors. A context-awareness system can be adopted for a seamless workflow by reflecting the user's intention with a depth camera or various sensors [12]. Enhanced augmented information or guides could be adapted by analyzing the user behavior, state of the prototype, or progress $[11,47]$. In addition, physical feedback or support could be applied. In the workbench, digital fabrication tools, such as a laser cutter, 3D printer, or robot arm, could be combined with the AR system to support the creation process [37, $23,24]$.

WIKA supports the creation process from design to making, but the initial ideation phase is not included in the system. During the creation process, ideas frequently modified according to the problem occurred in the programming and making phase. A generative design approach using the AR system could be applied $[15,17,8]$. For example, movement and interaction could be generated from the sketch of the user, and then the user can easily change and reflect the idea during the creation process.

There is still some work remaining to improve the system and study. Even though the participants created the diverse IKPF, there was a limitation in expression because of the limited types of sensors, motors, and mechanisms. It is required to provide various types of sensors, motors, and mechanisms to support the diverse scenarios. The modification of movement is limited to rotation and resizing. Editing or replacing the 
trajectory function is also necessary. The rule authoring interface can be improved for sophisticated programming. There are many conditions besides the min-max range in the sensor control. It might be possible to provide a specific interface according to the sensor type (e.g., gesture authoring interface with motion sensor, selection interface for the RGB color sensor). Moreover, the motor can express the inconstant movement by changing the speed. The servo motor can express the specific angle of the movement, and the linear motor can express the linear motion. Programming by the demonstration method can be applied to expand the input and output events. A sensor value is already recorded with physical sensors, and an additional interface can be applied to authoring the movement of motors in the physical environment. A more complex programming statement can be adopted with well-designed visualization and tangible objects. For instance, additional AR plate can represent the if-statement or counters. The AND and OR logic gates can be express by splitting the line. A thoughtful interface design for including many cases would be necessary for non-experts.

A more efficient and lightweight projected AR system would be effective to use in a physical environment. We applied the mobile device to bridge the workflow and support user activities with tangible objects and projected AR. Using the mobile device, it was possible to flexibly change the interface according to the user's intention without additional devices. However, some participants said that it was inconvenient to move the mobile device in the small workspace. A touch tabletop system can be used for the interface, but tangible objects will cover the interface. A well-defined gesture can be another option for providing an intuitive interface in the projected AR system. A hand-held AR device or mixed reality system with a head-mounted display has the potential to provide the programming and simulation in the $3 \mathrm{D}$ context. With advanced system, it might be possible to improve the quality of the outcome by supporting the $2.5 \mathrm{D}$ or 3D IKA.

WIKA, with an improved system, could be applied for educational purposes to provide training for engineering, computational, and artistic skills. To do so, additional exploration and improvement are necessary. Above all, a more structured user study for investigating the effects of physical and digital integration must be preceded.

\section{CONCLUSION}

In this paper, we presented WIKA, an integrated environment to explore and build the IKA. The projected AR system offers physical and digital integration with immediately visible results. The system features the combination of a tangible interface and augmented information that supports the whole creation process in a single workspace from the design to the making process. WIKA showed a tangible interaction technique for the interactivity definition and physical creation tasks using projected AR. The AR plates and the mobile device effectively connect the workflow and activities of the user. The user study revealed that WIKA facilitated a rapid and rich creation of the IKPF. We expect that this research will inspire the development of integrated systems that bridge the physi- cal and digital environment or support the creation, including movement and interactivity.

\section{ACKNOWLEDGMENTS}

This research was supported by the Global Ph.D. Fellowship Program through the National Research Foundation of Korea (NRF-2019H1A2A1074442).

\section{REFERENCES}

[1] 2012. TinkerCAD circuits. (2012). https://www.tinkercad.com/circuits accessed on 17 October 2019.

[2] Fraser Anderson, Tovi Grossman, and George Fitzmaurice. 2017. Trigger-Action-Circuits: Leveraging Generative Design to Enable Novices to Design and Build Circuitry. In Proceedings of the 30th Annual ACM Symposium on User Interface Software and Technology (UIST '17). ACM, New York, NY, USA, 331-342. DOI : http://dx.doi.org/10.1145/3126594.3126637

[3] Michelle Annett, Tovi Grossman, Daniel Wigdor, and George Fitzmaurice. 2015. MoveableMaker: facilitating the design, generation, and assembly of moveable papercraft. In Proceedings of the 28th Annual ACM Symposium on User Interface Software \& Technology. 565-574.

[4] Ayah Bdeir. 2009. Electronics as material: littleBits. In Proceedings of the 3rd International Conference on Tangible and Embedded Interaction. 397-400.

[5] Alvaro Cassinelli and Daniel Saakes. 2017. Data flow, spatial physical computing. In Proceedings of the Eleventh International Conference on Tangible, Embedded, and Embodied Interaction. 253-259.

[6] Duygu Ceylan, Wilmot Li, Niloy J Mitra, Maneesh Agrawala, and Mark Pauly. 2013. Designing and fabricating mechanical automata from mocap sequences. ACM Transactions on Graphics (TOG) 32, 6 (2013), $1-11$.

[7] Jeeyong Chung, Kyungeun Min, and Woohun Lee. 2013. CUBEMENT: Democratizing Mechanical Movement Design. In Proceedings of the 8th International Conference on Tangible, Embedded and Embodied Interaction (TEI '14). ACM, New York, NY, USA, 81-84. DOI : http://dx.doi.org/10.1145/2540930.2540933

[8] Stelian Coros, Bernhard Thomaszewski, Gioacchino Noris, Shinjiro Sueda, Moira Forberg, Robert W. Sumner, Wojciech Matusik, and Bernd Bickel. 2013. Computational Design of Mechanical Characters. ACM Trans. Graph. 32, 4, Article 83 (July 2013), 12 pages. DOI : http://dx. doi .org/10.1145/2461912.2461953

[9] Peter Dalsgaard and Kim Halskov. 2012. Tangible 3D tabletops: combining tangible tabletop interaction and 3D projection. In Proceedings of the 7th Nordic Conference on Human-Computer Interaction: Making Sense Through Design. 109-118. 
[10] Sean Follmer, David Carr, Emily Lovell, and Hiroshi Ishii. 2010. CopyCAD: Remixing Physical Objects with Copy and Paste from the Real World. In Adjunct Proceedings of the 23Nd Annual ACM Symposium on User Interface Software and Technology (UIST '10). ACM, New York, NY, USA, 381-382. DOI : http://dx.doi.org/10.1145/1866218.1866230

[11] Markus Funk, Thomas Kosch, and Albrecht Schmidt. 2016. Interactive worker assistance: comparing the effects of in-situ projection, head-mounted displays, tablet, and paper instructions. In Proceedings of the 2016 ACM International Joint Conference on Pervasive and Ubiquitous Computing. 934-939.

[12] Jun Gong, Fraser Anderson, George Fitzmaurice, and Tovi Grossman. 2019. Instrumenting and Analyzing Fabrication Activities, Users, and Expertise. In Proceedings of the 2019 CHI Conference on Human Factors in Computing Systems. 1-14.

[13] Valentin Heun, Shunichi Kasahara, and Pattie Maes. 2013. Smarter objects: using AR technology to program physical objects and their interactions. In CHI'13 Extended Abstracts on Human Factors in Computing Systems. 961-966.

[14] Michael S Horn and Robert JK Jacob. 2007. Designing tangible programming languages for classroom use. In Proceedings of the 1st international conference on Tangible and embedded interaction. 159-162.

[15] Yunwoo Jeong, Han-Jong Kim, and Tek-Jin Nam. 2018. Mechanism perfboard: An augmented reality environment for linkage mechanism design and fabrication. In Proceedings of the 2018 CHI Conference on Human Factors in Computing Systems. 1-11.

[16] Sergi Jordà, Günter Geiger, Marcos Alonso, and Martin Kaltenbrunner. 2007. The reacTable: exploring the synergy between live music performance and tabletop tangible interfaces. In Proceedings of the 1st international conference on Tangible and embedded interaction. 139-146.

[17] Seokbin Kang, Leyla Norooz, Virginia Byrne, Tamara Clegg, and Jon E Froehlich. 2018. Prototyping and simulating complex systems with paper craft and augmented reality: An initial investigation. In Proceedings of the Twelfth International Conference on Tangible, Embedded, and Embodied Interaction. 320-328.

[18] Majeed Kazemitabaar, Jason McPeak, Alexander Jiao, Liang He, Thomas Outing, and Jon E Froehlich. 2017. Makerwear: A tangible approach to interactive wearable creation for children. In Proceedings of the 2017 chi conference on human factors in computing systems. 133-145.

[19] Annie Kelly, R Benjamin Shapiro, Jonathan de Halleux, and Thomas Ball. 2018. ARcadia: A rapid prototyping platform for real-time tangible interfaces. In
Proceedings of the 2018 CHI Conference on Human Factors in Computing Systems. 1-8.

[20] Chang-Min Kim and Tek-Jin Nam. 2015. G-raff: an elevating tangible block for spatial tabletop interaction. In Proceedings of the 33rd Annual ACM Conference on Human Factors in Computing Systems. 4161-4164.

[21] Han-Jong Kim, Yunwoo Jeong, Ju-Whan Kim, and Tek-Jin Nam. 2018. A prototyping tool for kinetic mechanism design and fabrication: Developing and deploying M. Sketch for science, technology, engineering, the arts, and mathematics education. Advances in Mechanical Engineering 10, 12 (2018), 1687814018804104.

[22] Han-Jong Kim, Ju-Whan Kim, and Tek-Jin Nam. 2016. miniStudio: Designers' Tool for Prototyping Ubicomp Space with Interactive Miniature. In Proceedings of the 2016 CHI Conference on Human Factors in Computing Systems. 213-224.

[23] Jeeeun Kim, Haruki Takahashi, Homei Miyashita, Michelle Annett, and Tom Yeh. 2017. Machines as co-designers: A fiction on the future of human-fabrication machine interaction. In Proceedings of the 2017 CHI Conference Extended Abstracts on Human Factors in Computing Systems. 790-805.

[24] Jeeeun Kim, Clement Zheng, Haruki Takahashi, Mark D Gross, Daniel Ashbrook, and Tom Yeh. 2018. Compositional 3D printing: expanding \& supporting workflows towards continuous fabrication. In Proceedings of the 2nd ACM Symposium on Computational Fabrication. 1-10.

[25] Yoonji Kim, Youngkyung Choi, Hyein Lee, Geehyuk Lee, and Andrea Bianchi. 2019. VirtualComponent: A Mixed-Reality Tool for Designing and Tuning Breadboarded Circuits. In Proceedings of the 2019 CHI Conference on Human Factors in Computing Systems. $1-13$.

[26] Varsha Koushik, Darren Guinness, and Shaun K Kane. 2019. StoryBlocks: A Tangible Programming Game To Create Accessible Audio Stories. In Proceedings of the 2019 CHI Conference on Human Factors in Computing Systems. 1-12.

[27] Lego 1932. (1932). https://www.lego.com/en-us/technic.

[28] Nicola Leonardi, Marco Manca, Fabio Paternò, and Carmen Santoro. 2019. Trigger-action programming for personalising humanoid robot behaviour. In Proceedings of the 2019 CHI Conference on Human Factors in Computing Systems. 1-13.

[29] Jiahao Li, Jeeeun Kim, and Xiang'Anthony' Chen. 2019. Robiot: A Design Tool for Actuating Everyday Objects with Automatically Generated 3D Printable Mechanisms. In Proceedings of the 32nd Annual ACM Symposium on User Interface Software and Technology. 673-685. 
[30] Joanne Lo, Cesar Torres, Isabel Yang, Jasper O’Leary, Danny Kaufman, Wilmot Li, Mira Dontcheva, and Eric Paulos. 2016. Aesthetic electronics: Designing, sketching, and fabricating circuits through digital exploration. In Proceedings of the 29th Annual Symposium on User Interface Software and Technology. 665-676.

[31] Vittorio Megaro, Bernhard Thomaszewski, Damien Gauge, Eitan Grinspun, Stelian Coros, and Markus Gross. 2014. ChaCra: An Interactive Design System for Rapid Character Crafting. In Proceedings of the ACM SIGGRAPH/Eurographics Symposium on Computer Animation (SCA '14). Eurographics Association, Aire-la-Ville, Switzerland, Switzerland, 123-130. http://dl . acm.org/citation. cfm?id=2849517.2849538

[32] Vittorio Megaro, Jonas Zehnder, Moritz Bächer, Stelian Coros, Markus H Gross, and Bernhard Thomaszewski. 2017. A computational design tool for compliant mechanisms. ACM Trans. Graph. 36, 4 (2017), 82-1.

[33] Hyunjoo Oh, Jeeeun Kim, Cory Morales, Mark Gross, Michael Eisenberg, and Sherry Hsi. 2017. FoldMecha: Exploratory Design and Engineering of Mechanical Papercraft. In Proceedings of the Eleventh International Conference on Tangible, Embedded, and Embodied Interaction. 131-139.

[34] Hyunjoo Oh, Tung D Ta, Ryo Suzuki, Mark D Gross, Yoshihiro Kawahara, and Lining Yao. 2018. PEP (3D Printed Electronic Papercrafts): An Integrated Approach for 3D Sculpting Paper-Based Electronic Devices.. In CHI. 441.

[35] Eun Young Park. 2016. LINKKI: A planar linkage-based kinetic toy as a tool for education and design. In Proceedings of the 6th Annual Conference on Creativity and Fabrication in Education. 86-89.

[36] Esben Warming Pedersen and Kasper Hornbæk. 2011. Tangible bots: interaction with active tangibles in tabletop interfaces. In Proceedings of the SIGCHI Conference on Human Factors in Computing Systems. 2975-2984.

[37] Huaishu Peng, Jimmy Briggs, Cheng-Yao Wang, Kevin Guo, Joseph Kider, Stefanie Mueller, Patrick Baudisch, and François Guimbretière. 2018. RoMA: Interactive fabrication with augmented reality and a robotic 3D printer. In Proceedings of the 2018 CHI Conference on Human Factors in Computing Systems. 1-12.

[38] Hayes Solos Raffle, Amanda J. Parkes, and Hiroshi Ishii. 2004. Topobo: A Constructive Assembly System with Kinetic Memory. In Proceedings of the SIGCHI Conference on Human Factors in Computing Systems (CHI '04). ACM, New York, NY, USA, 647-654. DOI : http://dx.doi.org/10.1145/985692.985774

[39] Raf Ramakers, Kashyap Todi, and Kris Luyten. 2015. PaperPulse: an integrated approach for embedding electronics in paper designs. In Proceedings of the 33rd
Annual ACM Conference on Human Factors in Computing Systems. 2457-2466.

[40] Mitchel Resnick, John Maloney, Andrés Monroy-Hernández, Natalie Rusk, Evelyn Eastmond, Karen Brennan, Amon Millner, Eric Rosenbaum, Jay Silver, Brian Silverman, and Yasmin Kafai. 2009. Scratch: Programming for All. Commun. ACM 52, 11 (Nov. 2009), 60-67. D0I :

http://dx.doi.org/10.1145/1592761.1592779

[41] Peter Schmitt and Susanne Seitinger. 2009. Plywood punk: a holistic approach to designing animated artifacts. In Proceedings of the 3rd International Conference on Tangible and Embedded Interaction. 123-126.

[42] Science Box 1982. (June 1982). http://www.jeilscience.co.kr.

[43] Hariharan Subramonyam, Steven M Drucker, and Eytan Adar. 2019. Affinity Lens: Data-Assisted Affinity Diagramming with Augmented Reality. In Proceedings of the 2019 CHI Conference on Human Factors in Computing Systems. 1-13.

[44] Ryo Suzuki, Jun Kato, Mark D Gross, and Tom Yeh. 2018. Reactile: Programming Swarm User Interfaces through Direct Physical Manipulation. In Proceedings of the 2018 CHI Conference on Human Factors in Computing Systems. 1-13.

[45] Bernhard Thomaszewski, Stelian Coros, Damien Gauge, Vittorio Megaro, Eitan Grinspun, and Markus Gross. 2014. Computational Design of Linkage-based Characters. ACM Trans. Graph. 33, 4, Article 64 (July 2014), 9 pages. DOI :

http://dx.doi.org/10.1145/2601097.2601143

[46] John Underkoffler and Hiroshi Ishii. 1999. Urp: a luminous-tangible workbench for urban planning and design. In Proceedings of the SIGCHI conference on Human Factors in Computing Systems. 386-393.

[47] Bret Victor. 2014. Seeing spaces. In Talk at EG conference.

[48] Christian Weichel, Manfred Lau, David Kim, Nicolas Villar, and Hans W. Gellersen. 2014. MixFab: A Mixed-reality Environment for Personal Fabrication. In Proceedings of the 32Nd Annual ACM Conference on Human Factors in Computing Systems (CHI '14). ACM, New York, NY, USA, 3855-3864. DOI : http://dx.doi . org/10.1145/2556288.2557090

[49] Yunbo Zhang, Wei Gao, Luis Paredes, and Karthik Ramani. 2016. Cardboardizer: Creatively customize, articulate and fold $3 \mathrm{~d}$ mesh models. In Proceedings of the 2016 CHI Conference on Human Factors in Computing Systems. 897-907.

[50] Lifeng Zhu, Weiwei Xu, John Snyder, Yang Liu, Guoping Wang, and Baining Guo. 2012. Motion-guided mechanical toy modeling. ACM Transactions on Graphics (TOG) 31, 6 (2012), 1-10. 